\title{
Identification of novel antifungal agents: antimicrobial evaluation, SAR, ADME-Tox and molecular docking studies of a series of imidazole derivatives
}

\author{
Btissam Bouchal ${ }^{1 \dagger}$, Farid Abrigach ${ }^{2 \dagger}$, Abdelilah Takfaoui ${ }^{2}$, Manal Elidrissi Errahhali ${ }^{1}$, Mounia Elidrissi Errahhali ${ }^{1}$, \\ Pierre H. Dixneuf ${ }^{3}$, Henri Doucet ${ }^{3}$, Rachid Touzani ${ }^{2}$ and Mohammed Bellaoui ${ }^{1 *}$ (0)
}

\begin{abstract}
Thirty-four imidazole-based compounds synthesized by one-pot catalytic method were evaluated for their antifungal and antibacterial activities against several fungal and bacterial strains. None of the compounds had antibacterial activity. Interestingly, compounds 1, 2, 3, 10 and $\mathbf{1 5}$ displayed a strong antifungal activity against all the tested fungal species, while compounds $\mathbf{5}, \mathbf{7}, \mathbf{9}, \mathbf{1 1}, \mathbf{2 1}$ and $\mathbf{2 7}$ showed a moderate antifungal activity. To better understand the biological activity of the most active compounds ADME-Tox and molecular docking studies were carried out. Interestingly, compounds $\mathbf{1}, \mathbf{2}, \mathbf{3}, \mathbf{7}, \mathbf{1 0}$ and $\mathbf{1 5}$ showed excellent bioavailability. In addition, compounds $\mathbf{1}, \mathbf{2}$ and $\mathbf{3}$, exhibited good toxicity profiles. Docking studies of the two most active compounds $\mathbf{2}\left(\mathrm{IC}_{50}\right.$ of $\left.95 \pm 7.07 \mu \mathrm{M}\right)$ and $\mathbf{1 0}\left(\mathrm{I} \mathrm{C}_{50}\right.$ of $235 \pm 7.07 \mu \mathrm{M})$ suggested that they might act by inhibiting the fungal lanosterol 14a-demethylase. Therefore, these novel antifungal agents merit further characterization for the development of new antifungal therapeutics.
\end{abstract}

Keywords: Imidazole, Antifungal, Antibacterial, Structure-activity relationship, ADME-Tox, Docking

\section{Introduction}

Human infectious diseases are among the top ten causes of death worldwide, according to the World Health Organization [1]. Therefore, infectious diseases are among the most important public health problems around the world, especially in developing countries [2]. Drug-resistant infections are also a growing public health threat worldwide [3-5]. As a matter of fact, the increasing number of antifungal drug resistance has become more frequent and serious, creating a need for safer and more effective antifungal therapies [6-9]. Indeed, increasing resistance of fungal pathogens to current antifungal drugs is one of the reasons for the difficulty to tackle fungal infections, particularly in immune-compromised

\footnotetext{
*Correspondence: bmbellaoui@gmail.com

${ }^{\dagger}$ Btissam Bouchal and Farid Abrigach contributed equally to this work

${ }^{1}$ Genetics Unit, Faculty of Medicine and Pharmacy of Oujda, University Mohammed Premier, Oujda, Morocco

Full list of author information is available at the end of the article
}

individuals [10]. Given this difficulty in controlling fungal infections, a new class of antifungal drugs with novel mechanisms of action and broad spectrum of activity must be discovered.

In this view, many heterocyclic compounds have been studied in order to discover novel antimicrobial agents. In fact, heterocyclic chemistry has grown considerably and more than $90 \%$ of new drugs contain heterocycles [11]. More particularly, imidazole-based heterocyclic compounds occupy a prominent place in heterocyclic chemistry $[12,13]$. Members of this family of compounds have diverse biological, pharmacological, environmental and industrial applications [14]. Indeed, this type of compounds act as inhibitors of p38 MAP kinase and B-Raf [15], and showed anti-inflammatory, anti-cancer, antifungal, anti-tuberculosis, and anti-diabetic activities [16-18]. Accordingly, several imidazole-based heterocyclic compounds have been clinically used to treat many diseases such as Bifonazole which is a clinically used antifungal 
agent with a broad spectrum of activity [19, 20], Metronidazole used to treat a wide variety of bacterial and parasitic infections [21], Cimetidine used in the treatment of duodenal and gastric ulcers [22], and Eprosartan used as angiotensin II inhibitor and antihypertensive agent [23].

In this context, we sought to study the antifungal and antibacterial biological activities of a series of 34 imidazole-based heterocyclic compounds that have been synthesized by a simple one-pot catalytic method. We have thus identified five imidazole derivatives 1, 2, 3, 10 and 15, which act specifically as potent antifungal agents and lack antibacterial activity. Their structure-activity relationship analysis (SAR), ADME-Tox profiles, and molecular docking studies were carried out to understand their biological activity. These novel antifungal agents merit further characterization and can serve as a promising lead compounds for the discovery of new antifungal therapeutics.

\section{Results and discussion}

The imidazole-based compounds studied in this paper were synthesized by one-pot catalytic method [24] and are shown in Fig. 1. The spectroscopic information of these compounds is described below in materials and methods.

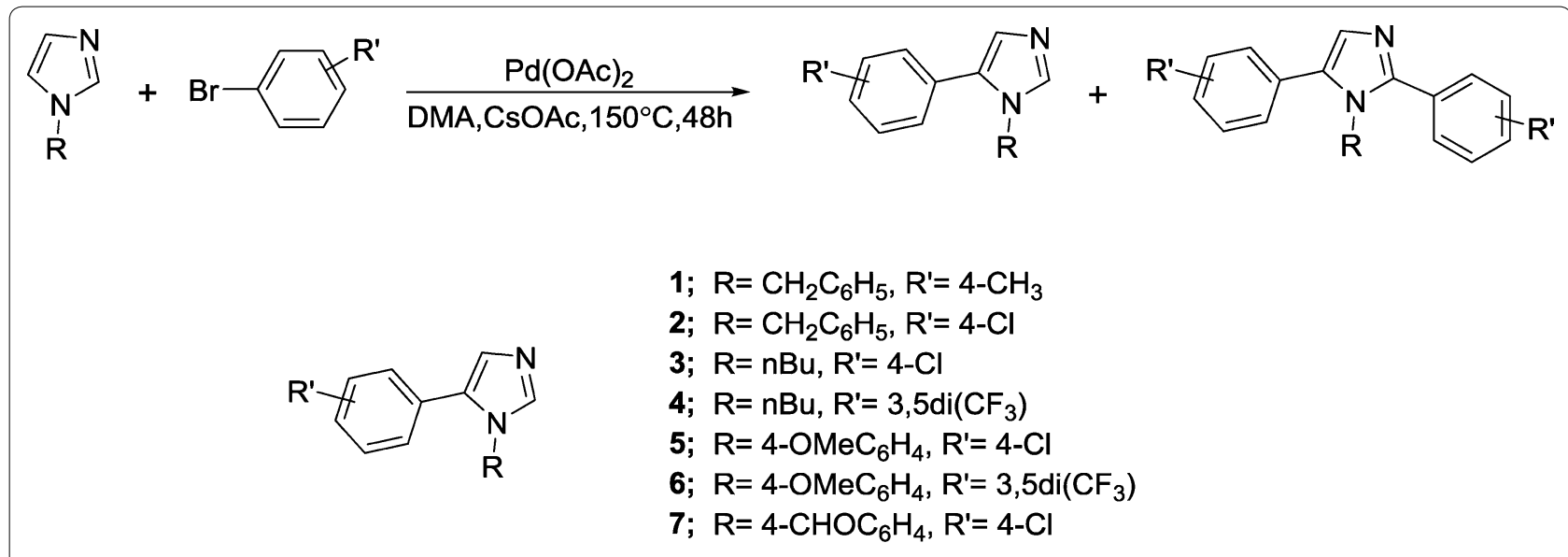<smiles>[R]c1ccc(-c2cnc(-c3ccc([R])cc3)n2[R])cc1</smiles>

8; $\mathrm{R}=\mathrm{CH}_{3}, \mathrm{R}^{\prime}=\mathrm{H}$

9; $\mathrm{R}=\mathrm{CH}_{3}, \mathrm{R}^{\prime}=4-\mathrm{CH}_{3}$

10; $\mathrm{R}=\mathrm{CH}_{3}, \mathrm{R}^{\prime}=3-\mathrm{CH}_{3}$

11; $\mathrm{R}=\mathrm{CH}_{3}, \mathrm{R}^{\prime}=2-\mathrm{CH}_{3}$

12; $\mathrm{R}=\mathrm{CH}_{3}, \mathrm{R}^{\prime}=4-\mathrm{NC}$

13; $\mathrm{R}=\mathrm{CH}_{3}, \mathrm{R}^{\prime}=3-\mathrm{NC}$

14; $\mathrm{R}=\mathrm{CH}_{3}, \mathrm{R}^{\prime}=4-\mathrm{F}$

15; $\mathrm{R}=\mathrm{CH}_{3}, \mathrm{R}^{\prime}=2-\mathrm{F}$

16; $\mathrm{R}=\mathrm{CH}_{3}, \mathrm{R}^{\prime}=3-\mathrm{CF}_{3}$

17; $\mathrm{R}=\mathrm{CH}_{3}, \mathrm{R}^{\prime}=4-\mathrm{Cl}$

27; $\mathrm{R}=\mathrm{CH}_{3}, \mathrm{R}^{\prime}=\mathrm{H}$

28; $\mathrm{R}=\mathrm{CH}_{2} \mathrm{C}_{6} \mathrm{H}_{5}, \mathrm{R}^{\prime}=\mathrm{H}$

29; $\mathrm{R}=\mathrm{nBu}, \mathrm{R}^{\prime}=\mathrm{H}$

30; $\mathrm{R}=4-\mathrm{CHOC}_{6} \mathrm{H}_{4}, \mathrm{R}^{\prime}=\mathrm{H}$

31; $\mathrm{R}=4-\mathrm{OMeC}_{6} \mathrm{H}_{4}, \mathrm{R}^{\prime}=\mathrm{H}$

32; $\mathrm{R}=\mathrm{CH}_{3}, \mathrm{R}^{\prime}=3,5 \mathrm{di}\left(\mathrm{CF}_{3}\right) \mathrm{C}_{6} \mathrm{H}_{3}$

33; $\mathrm{R}=\mathrm{nBu}, \mathrm{R}^{\prime}=3,5 \mathrm{di}\left(\mathrm{CF}_{3}\right) \mathrm{C}_{6} \mathrm{H}_{3}$

34; $\mathrm{R}=\mathrm{CH}_{2} \mathrm{C}_{6} \mathrm{H}_{5}, \mathrm{R}^{\prime}=3,5 \mathrm{di}\left(\mathrm{CF}_{3}\right) \mathrm{C}_{6} \mathrm{H}_{3}$
18; $\mathrm{R}=\mathrm{CH}_{3}, \mathrm{R}^{\prime}=4-\mathrm{NO}_{2}$

19; $\mathrm{R}=\mathrm{CH}_{3}, \mathrm{R}^{\prime}=3-\mathrm{NO}_{2}$

20; $\mathrm{R}=\mathrm{CH}_{3}, \mathrm{R}^{\prime}=4-\mathrm{OMe}$

21; $\mathrm{R}=\mathrm{CH}_{3}, \mathrm{R}^{\prime}=4-\mathrm{CHO}$

22; $\mathrm{R}=\mathrm{CH}_{3}, \mathrm{R}^{\prime}=4-\mathrm{COMe}$

23; $\mathrm{R}=\mathrm{CH}_{3}, \mathrm{R}^{\prime}=4-\mathrm{COEt}$

24; $\mathrm{R}=\mathrm{CH}_{2} \mathrm{C}_{6} \mathrm{H}_{5}, \mathrm{R}^{\prime}=4-\mathrm{Cl}$

25; $\mathrm{R}=\mathrm{nBu}, \mathrm{R}^{\prime}=4-\mathrm{CH}_{3}$

26; $R=n B u, R^{\prime}=4-C l$<smiles>[R]c1nc(-c2cc(C(F)(F)F)cc(C(F)(F)F)c2)n([R])c1-c1cc(C(F)(F)F)cc(C(F)(F)F)c1</smiles>

Fig. 1 Synthetic route and structure of the molecules studied in this paper 


\section{Antifungal and antibacterial activities of imidazole derivatives}

We first evaluated the antifungal activity of our imidazole derivatives against three fungal species (S. cerevisiae, $C$. albicans and C. krusei) as described in materials and methods. All the compounds were used at $500 \mu \mathrm{M}$. Interestingly, compounds 1, 2, 3, 10 and 15 displayed strong antifungal activity (greater than $80 \%$ growth inhibition) against all three tested fungal species, whereas compounds 5, 7, 9, 11, 21 and 27 showed moderate antifungal activity (20-50\% growth inhibition) (Fig. 2). On the other hand, compounds, $6,8,16,17,25,31$ exhibited weak antifungal activity (5-20\% growth inhibition), while the rest of the molecules were not toxic to yeast cells (Fig. 2).

Regarding the antibacterial activity, all the compounds were tested for toxicity against three gram-negative bacterial strains (E. coli, C. freundii and S. braenderup) and two gram-positive bacterial strains (L. monocytogenes and $S$. aureus). All the compounds showed no antibacterial activity against all bacterial strains used. Therefore, to better understand the antifungal activity of the most active compounds, SAR, ADME-Tox and molecular docking studies were carried out and are detailed below.

\section{SAR of imidazole derivatives}

The SAR analysis of the mono-arylated series (1-7) revealed that the antifungal activity of these compounds depends essentially on the attached group (R) at the position 1 of imidazole moiety and the attached group (R') of the phenyl ring. Investigation of $\mathrm{R}$ moiety in compounds $\mathbf{2}$, 3, 5 and 7, showed that the presence of $\mathrm{OMeC}_{6} \mathrm{H}_{4}$ substituent in compound 5 or $\mathrm{CHOC}_{6} \mathrm{H}_{4}$ group in compound 7 resulted in moderate antifungal activity. Whereas, the presence of benzyl substituent in compound $\mathbf{2}$ or $n$-Butyl group in compound 3 resulted in high antifungal activity. Further evaluation of compounds $\mathbf{2}$ and $\mathbf{3}$ demonstrated that $\mathbf{2}$ was more potent than 3 , with a half-maximal inhibitory concentration $\left(\mathrm{IC}_{50}\right)$ against $S$. cerevisiae of $95 \pm 7.07 \mu \mathrm{M}$ and $220 \pm 14.14 \mu \mathrm{M}$, respectively (Table 1 ).

These findings suggest that the small size and the electron withdrawing character of the substituent at the position 1 of imidazole moiety are important for the antifungal activity of these compounds. Regarding the comparison between compounds $\mathbf{1}$ and 2, we observed a stronger antifungal activity against $S$. cerevisiae with $\mathbf{2}\left(\mathrm{IC}_{50}\right.$ of $95 \pm 7.07 \mu \mathrm{M})$ compared to $\mathbf{1}\left(\mathrm{IC}_{50}\right.$ of $\left.240 \pm 14.14 \mu \mathrm{M}\right)$, suggesting that the replacement of chlorine (an electron withdrawing group) with methyl (an electron donating group) decreases the antifungal potential. Together, these data suggest that the introduction of electron withdrawing substituents in R and R' increases the antifungal activity of these compounds.

The SAR analysis of the 2, 5-diarylated imidazole derivatives series $(8-26)$ revealed that the introduction of electron withdrawing or electron donating groups into the phenyl rings at the para position resulted in moderate antifungal activity $(9,21)$ or no activity $(12,14,17,18$, 20, 22 and 23). We also observed that the presence of electron withdrawing groups $\left[\mathrm{CF}_{3}(\mathbf{1 6}), \mathrm{CN}(\mathbf{1 3})\right.$ or $\mathrm{NO}_{2}$ (19)] into the phenyl rings at the meta position resulted in loss of antifungal activity. However, the presence of an electron donating group (methyl group) at the meta position of the phenyl rings (10) resulted in strong antifungal activity. We also found that the substitution of the phenyl rings at the ortho position with Fluor or methyl groups led to strong antifungal activity (15) or moderate activity (11). Further evaluation of the most active compounds of this series (10 and 15) has demonstrated that compound 10 was more potent than compound 15 , with $\mathrm{IC}_{50}$ 's against $S$. cerevisiae of $235 \pm 7.07 \mu \mathrm{M}$ and $305 \pm 21.21 \mu \mathrm{M}$, respectively (Table 1). Together, these findings suggest that the antifungal activity of these diarylated imidazole derivatives depends on the size (small or bulky), position (para, ortho or meta) and electronic effect (withdrawing or donating) of the substituent at the phenyl rings.

The SAR studies of the imidazole derivatives series (27 to 34) revealed that the presence of many strong electron withdrawing $\mathrm{CF}_{3}$ groups at the meta position of phenyl groups did not lead to any antifungal activity. Similarly, the introduction of bulky groups at the position 1 of imidazole moiety did not lead to significant growth inhibition against the tested fungal strains. These findings are consistent with the above observations which suggest that the presence of bulky substituent at the position 1 of imidazole moiety, or at the meta position of phenyl group is unfavorable for the antifungal activity.

\section{ADME-Tox predictions}

Currently, the computational predictions of pharmacokinetic and pharmacodynamic parameters such as absorption, distribution, metabolism excretion (ADME) and toxicity risks (Tox) are of great importance in the drug

(See figure on next page.)

Fig. 2 Antifungal activity of the studied compounds against Saccharomyces cerevisiae, Candida albicans and Candida krusei. Cells were cultured in the presence of $500 \mu \mathrm{M}$ of each compound for $24 \mathrm{~h}$ and growth rate was then assayed by the OD 600 . Growth in the presence of compound was expressed as a percentage relative to the untreated control. All experiments were carried out in triplicate and means were calculated \pm SD. ${ }^{*} p<0.05$ versus untreated control 

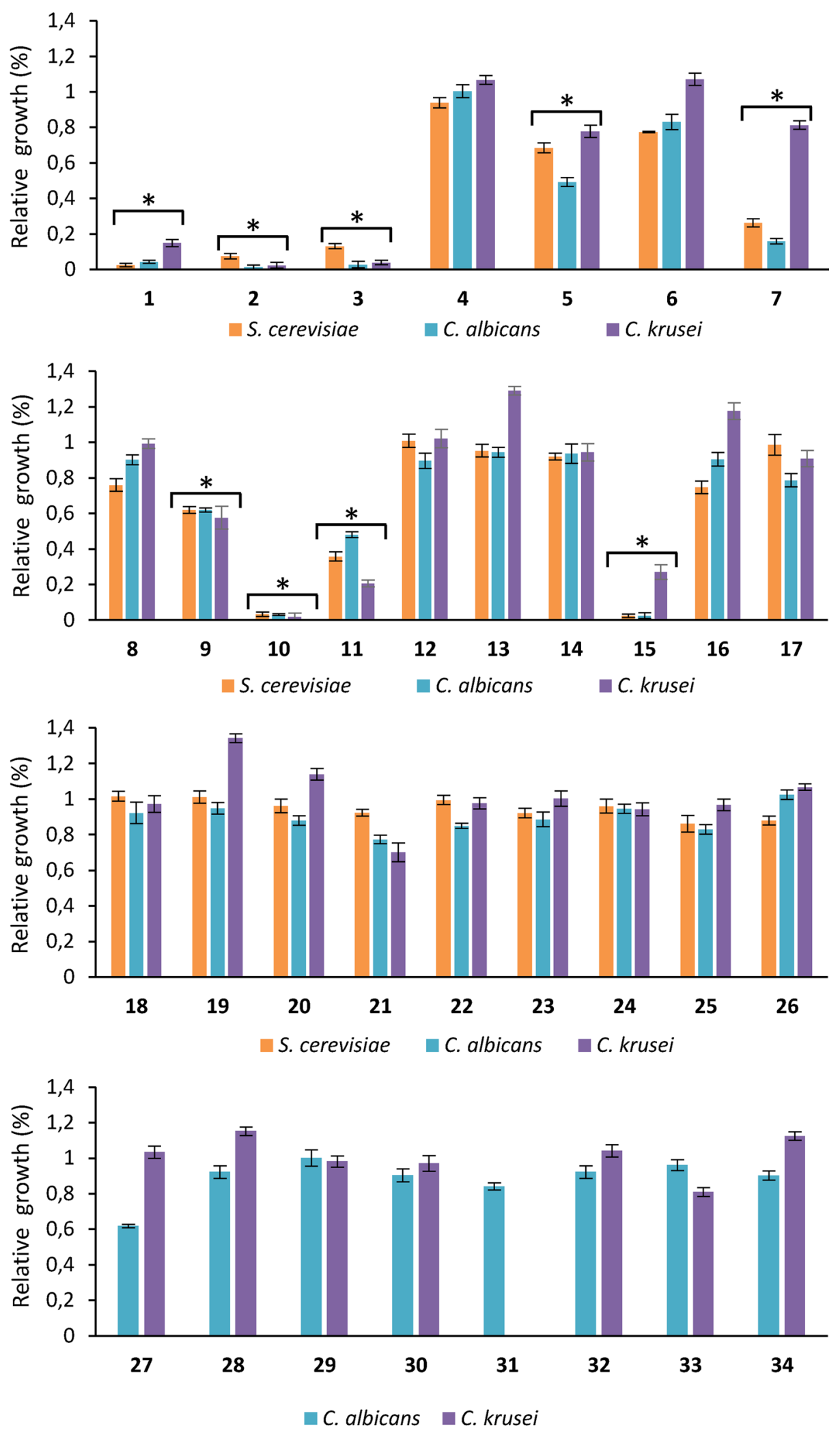
Table $1 \mathrm{IC}_{50}$ against $S$. cerevisiae of the imidazole derivatives with strong antifungal activity

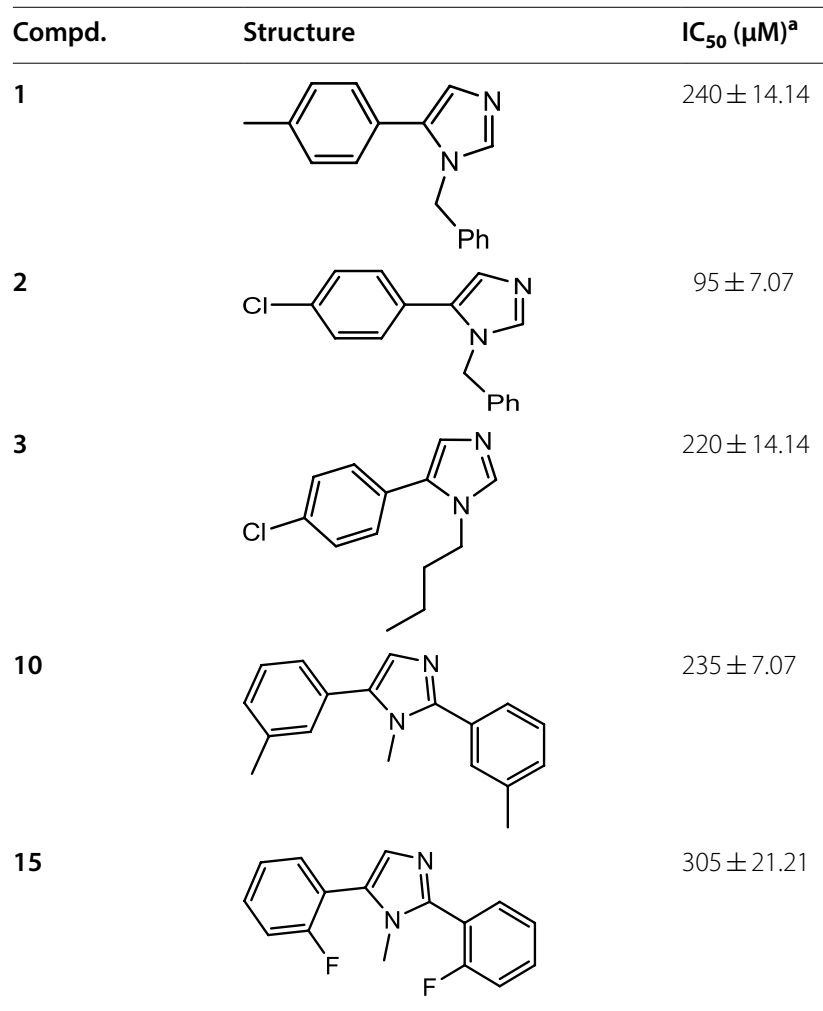

a The value obtained for each compound represents the mean of three independent experiments $\pm S D$

discovery process [25]. Therefore, in this study we used Molinspiration and DataWarroir programs, as described in Materials and Methods, to determine the ADME-Tox profiles of the most active compounds $(1,2,3,7,10,11$ and 15) as well as the reference antifungal drug Fluconazole.
As shown in Table 2, in silico prediction of toxicity properties (Mutagenic; Tumorigenic; Irritant; Reproductive effect) of our imidazole derivatives revealed that compounds 1, 2 and $\mathbf{3}$ have a good toxicological profile with no risk of mutagenicity, tumorigenicity, irritation or reproduction. These Tox properties are similar to those of Fluconazole. However, compound $\underline{7}$ showed a high toxic effect on reproduction probably due to the presence of the $\mathrm{C}=\mathrm{O}$ carbonyl group at the aldehyde moiety. Regarding 2, 5-diarylated imidazole derivatives 10, 11 and 15, toxicity prediction revealed that these compounds have a low mutagenic effect, but without risk of tumorigenicity, irritation or reproduction. Overall, these Tox studies suggest that compounds 1, 2 and 3 do not have any undesirable moieties involved in toxicity problems, while compounds 10, 11 and 15 exhibit acceptable toxicity profiles with a low mutagenic effect.

As stated above, Molinspiration was used to determine pharmacokinetic parameters of the most active compounds. Therefore, the following ADME characteristics were calculated: $n$-octanol/water partition coefficient characterizing lipophilicity $(\log P)$, molecular weight expressed in Daltons (MW), number of hydrogen bond acceptors $(n \mathrm{OH})$, number of hydrogen bond donors $(n \mathrm{OHNH})$, number of rotatable bonds (nrotb), and total polar surface area (TPSA). As shown in Table 2, all the studied compounds exhibit ADME characteristics that are in agreement with Lipinski's rule of five, which evaluate the drug-likeness, absorption and intestinal permeability of a compound [26, 27]. Indeed, for all the compounds $\log P$ are less than $5.0, \mathrm{MW}$ are less than $500, n \mathrm{OH}$ are less than $10, n \mathrm{OHNH}$ are less than 5 , nrotb are less than 10 and TPSA are less than $140 \AA^{2}$. Together, these data suggest that these studied

Table 2 Toxicity risks and physicochemical properties of the imidazole derivatives with good antifungal activity and the reference drug Fluconazole

\begin{tabular}{|c|c|c|c|c|c|c|c|c|c|c|}
\hline \multirow[t]{2}{*}{ Compound } & \multicolumn{4}{|c|}{ Toxicity risks } & \multicolumn{6}{|c|}{ Physicochemical properties } \\
\hline & MU & TU & IR & RE & $\log P$ & MW & $n \mathrm{OH}$ & nOHNH & nrotb & TPSA $\left(\AA^{2}\right)$ \\
\hline 1 & None & None & None & None & 3.88 & 248.33 & 2 & 0 & 3 & 17.83 \\
\hline 2 & None & None & None & None & 3.97 & 268.75 & 2 & 0 & 3 & 17.83 \\
\hline 3 & None & None & None & None & 3.57 & 234.73 & 2 & 0 & 4 & 17.83 \\
\hline 7 & None & None & None & High & 3.62 & 282.73 & 3 & 0 & 3 & 34.90 \\
\hline 10 & Low & None & None & None & 4.70 & 262.36 & 2 & 0 & 2 & 17.83 \\
\hline 11 & Low & None & None & None & 4.70 & 262.36 & 2 & 0 & 2 & 17.83 \\
\hline 15 & Low & None & None & None & 3.96 & 270.28 & 2 & 0 & 2 & 17.83 \\
\hline Fluconazole & None & None & None & None & 0.56 & 306.28 & 7 & 1 & 5 & 81.66 \\
\hline
\end{tabular}

MU, mutagenic; TU, tumorigenic; IR, irritant; RE, reproductive effect; LogP, octanol/water partition coefficient characterizing lipophilicity; MW, molecular weight expressed in Daltons; $n \mathrm{OH}$, number of hydrogen bond acceptors; $n \mathrm{OHNH}$, number of hydrogen bond donors; nrotb, number of rotatable bonds; TPSA, total polar surface area 
compounds present good bioavailability and therefore can be qualified as a good lead.

\section{Molecular docking studies}

Fungal lanosterol $14 \alpha$-demethylase (CYP51) is an attractive therapeutic target for the development of antifungal drugs $[28,29]$. This enzyme catalyzes an essential step in the synthesis of ergosterol which is an essential component of fungal cell membrane. CYP51 is the target of azoles, the most popular class of antifungal drugs. There has been a considerable amount of research interest into this enzyme and the azoles because of the dramatically increasing number of drug resistance among certain fungal species [30]. Ketoconazole is an antifungal imidazole that belongs to the azole class and it is currently used to treat a wide variety of fungal infections. Like other azoles, it acts by inhibiting selectively [31]. However, the use of

\section{Table 3 Docking analysis of some imidazole derivatives and the reference drug Fluconazole against Saccharomyces cerevisiae CYP51}

\begin{tabular}{llll}
\hline Compound & $\boldsymbol{\Delta G}_{\text {binding }}(\mathbf{k c a l} / \mathbf{m o l})$ & H-bond & Distance (̊̊) \\
\hline $\mathbf{2}$ & -6.857 & $\mathrm{~N}(3)-$ Cys470 & 2.31 \\
$\mathbf{1 0}$ & -6.791 & $\mathrm{~N}(3)-$ Arg489 & 2.41 \\
$\mathbf{6}$ & -5.616 & - & - \\
$\mathbf{3 0}$ & - & - & - \\
Fluconazole & -7.337 & $\mathrm{~N}(4)$-Arg467 & 2.70 \\
\hline
\end{tabular}

Ketoconazole has been limited because it is associated with clinically important toxic side effects $[32,33]$. Therefore, it is important to discover novel antifungal imidazoles which act by inhibiting selectively CYP51 without toxic side effects.

Considering the fact that our compounds have imidazole moiety we sought to study the possible binding of our most potent compounds to CYP51 as a possible target protein. Therefore, molecular docking studies were conducted in order to explore the affinity of our most potent imidazole derivatives towards CYP51 from $S$. cerevisiae. Molecular docking is a very powerful computational method for predicting and modeling the interactions between a small molecule and a protein target at the atomic level. Indeed, molecular docking is an important tool, which is widely used in drug design [34, 35].

Table 3 and Fig. 3 show the docking analysis results of compound $\mathbf{2}$ (the most potent compounds of the mono-arylated series), compound $\mathbf{1 0}$ (the most potent compounds of the 2,5-diarylated imidazole derivatives series), and the antifungal reference drug fluconazole towards CYP51 from S. cerevisiae.

Three parameters were used for docking analysis: the binding affinity expressed in $\mathrm{kcal} / \mathrm{mol}$, the interactions between ligand atoms and amino acid residues of the target protein, and the distance of these interactions. Therefore, our results of docking showed that the two tested imidazole derivatives (2 and 10) and fluconazole perform a hydrogen bond interaction ( $\mathrm{H}$-bond) between one of

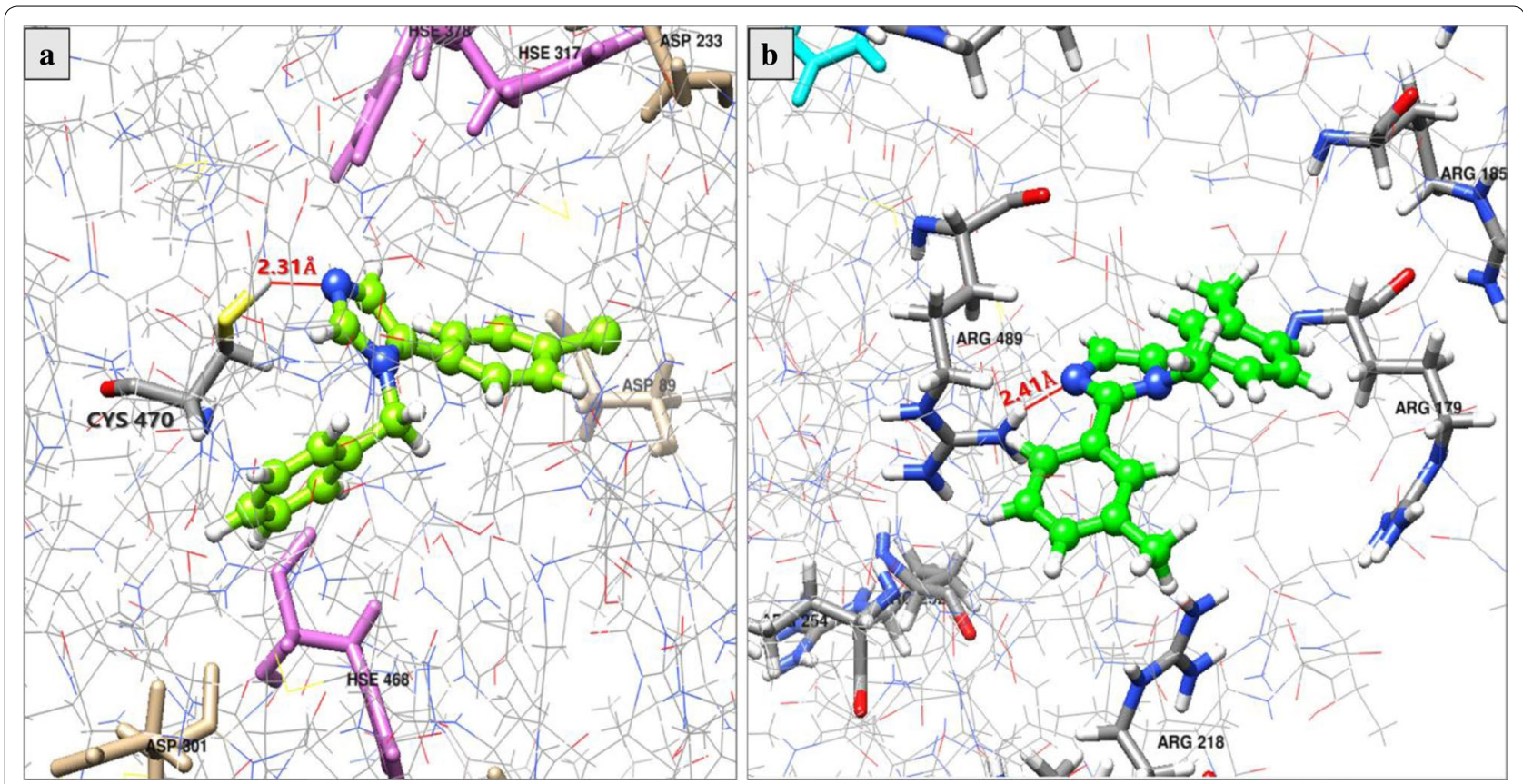

Fig. 3 Binding mode of compounds $\mathbf{2}$ (a) and $\mathbf{1 0}$ (b) with target enzyme CYP51 from S. cerevisiae 
their nitrogen atoms and an amino acid residue of the target protein. For fluconazole, nitrogen atom in position 4 makes one H-bond with Arg467 amino acid of the target protein at a bond distance of $2.70 \AA$ and a binding affinity of $-7.337 \mathrm{kcal} / \mathrm{mol}$. While for compound 2, nitrogen atom in position 3 makes one $\mathrm{H}$-bond with Cys470 amino acid of the target protein at a bond distance of $2.31 \AA$ and a binding affinity of $-6.857 \mathrm{kcal} / \mathrm{mol}$. Similarly, nitrogen atom in position 3 of compound $\mathbf{1 0}$ makes one $\mathrm{H}$-bond, but with Arg489 amino acid of the target protein with a bond distance of $2.41 \AA$ and a binding affinity of $-6.791 \mathrm{kcal} / \mathrm{mol}$.

To affirm correlation between in silico prediction of binding affinity to CYP51 and antifungal activity, we also docked two imidazole derivatives which lack antifungal activity (6 and 30) against CYP51 (Table 3). Interestingly, our docking studies revealed that compound $\mathbf{3 0}$ does not have binding affinity toward CYP51. Regarding compound 6, the results of docking indicated that this compound have lower binding affinity $(-5.616 \mathrm{kcal} / \mathrm{mol})$ toward CYP51 as compared to the active compounds 2 and $10(-6.857$ and $-6.791 \mathrm{kcal} / \mathrm{mol})$. Moreover, the docking result revealed that, like compound 30, compound $\mathbf{6}$ does not interact with CYP51 by hydrogen bond interaction. Together, these docking studies agreed with the antifungal activity and suggest that the two most active compounds $\mathbf{2}$ and $\mathbf{1 0}$ have good binding affinity with target enzyme CYP51 and therefore might act by inhibiting the fungal lanosterol $14 \alpha$-demethylase.

\section{Conclusion}

In this paper we investigated the antifungal and antibacterial biological activities of thirty-four imidazole-based compounds synthesized by one-pot catalytic method. Antifungal activity was assayed against five fungal species, while antibacterial activity was tested against five bacterial species. None of the tested compounds showed an antibacterial activity. Interestingly, compounds 1, 2, 3, $\mathbf{1 0}$ and $\mathbf{1 5}$ displayed a strong antifungal activity against all the tested fungal species, while compounds $5,7,9,11$, 21 and 27 showed a moderate antifungal activity. SAR studies revealed that the antifungal activity of these imidazole derivatives depends on the size (small or bulky), position (para, ortho or meta) and electronic effect (withdrawing or donating) of the substituents at the phenyl rings, as well as the bulkiness and the electronic effect of the substituent in position 1 of the imidazole moiety. ADME analysis showed that compounds 1, 2, 3, 7, 10 and 15 have excellent bioavailability. In addition, Tox studies showed that compounds 1, 2 and 3, have good toxicity profiles, whereas 10, 11 and 15 have low mutagenic effect. On the other hand, compound 7 is likely to cause toxicity because of the high toxic effect on reproduction.
Docking studies of the two most active compounds 2 and 10 suggested that they might act by inhibiting the fungal lanosterol $14 \alpha$-demethylase. Therefore, these novel antifungal agents merit further characterization and can serve as promising lead compounds for the discovery of new antifungal therapeutics.

\section{Materials and methods Chemistry}

The 34 imidazole-based molecules analyzed in this paper have been synthesized by a simple one-pot catalytic method [24]. The spectroscopic information of these compounds is as follows:

1-Benzyl-5-p-tolyl-1H-imidazole (1) $31 \%$ yield. ${ }^{1} \mathrm{H}$ NMR $\left(400 \mathrm{MHz}, \mathrm{CDCl}_{3}\right) \delta 7.48(\mathrm{~s}, 1 \mathrm{H}), 7.30-7.15(\mathrm{~m}$, $3 \mathrm{H}), 7.10(\mathrm{~s}, 4 \mathrm{H}), 7.04(\mathrm{~s}, 1 \mathrm{H}), 6.95(\mathrm{~d}, J=8.0 \mathrm{~Hz}, 2 \mathrm{H})$, $5.06(\mathrm{~s}, 2 \mathrm{H}), 2.29$ (s, 3H) ${ }^{13} \mathrm{C}$ NMR $\left(100 \mathrm{MHz}, \mathrm{CDCl}_{3}\right)$ $\delta 138.4,138.0,136.9,133.5,129.4,128.9,128.8,128.0$, $127.9,126.8,126.7,48.7,21.2$.

1-benzyl-5-(4-chlorophenyl)-1H-imidazole (2) $44 \%$ yield. ${ }^{1} \mathrm{H}$ NMR $\left(400 \mathrm{MHz}, \mathrm{CDCl}_{3}\right) \delta 7.51(\mathrm{~s}, 1 \mathrm{H}), 7.28$ $7.15(\mathrm{~m}, 5 \mathrm{H}), 7.12(\mathrm{~d}, J=8.4 \mathrm{~Hz}, 2 \mathrm{H}), 7.06(\mathrm{~s}, 1 \mathrm{H}), 6.92(\mathrm{~d}$, $J=8.4 \mathrm{~Hz}, 2 \mathrm{H}), 5.06(\mathrm{~s}, 2 \mathrm{H}) .{ }^{13} \mathrm{C}$ NMR $\left(100 \mathrm{MHz}, \mathrm{CDCl}_{3}\right)$ $\delta 139.0,136.5,134.2,132.3,130.1,129.0,128.9,128.6$, 128.2, 128.1,126.5, 48.8 .

1-butyl-5-(4-chlorophenyl)-1H-imidazole (3) $45 \%$ yield. ${ }^{1} \mathrm{H}$ NMR $\left(400 \mathrm{MHz}, \mathrm{CDCl}_{3}\right) \delta 7.50(\mathrm{~s}, 1 \mathrm{H}), 7.34$ (d, $J=8.4 \mathrm{~Hz}, 2 \mathrm{H}), 7.23(\mathrm{~d}, J=8.4 \mathrm{~Hz}, 2 \mathrm{H}), 6.98(\mathrm{~s}, 1 \mathrm{H})$, 3.87 (t, $J=7.5 \mathrm{~Hz}, 2 \mathrm{H}$ ), 1.53 (quint., $J=7.5 \mathrm{~Hz}, 2 \mathrm{H}$ ), 1.16 (sext., $J=7.5 \mathrm{~Hz}, 2 \mathrm{H}), 0.77$ (t, $J=7.5 \mathrm{~Hz}, 3 \mathrm{H}) .{ }^{13} \mathrm{C} \mathrm{NMR}$ $\left(100 \mathrm{MHz}, \mathrm{CDCl}_{3}\right) \delta 138.3,134.0,131.7,130.0,129.0$, 128.7, 128.3, 45.1, 32.9, 19.6, 13.4 .

5-(3,5-bis(trifluoromethyl)phenyl)-1-butyl-1H-imidazole (4) The product was obtained as trace observed by GC/ MS analysis of the crude mixture.

5-(4-chlorophenyl)-1-(4-methoxyphenyl)-1H-imidazole (5) $62 \%$ yield. ${ }^{1} \mathrm{H}$ NMR $\left(400 \mathrm{MHz}, \mathrm{CDCl}_{3}\right) \delta 7.57$ (s, $1 \mathrm{H}), 7.16(\mathrm{~d}, J=8.4 \mathrm{~Hz}, 2 \mathrm{H}), 7.13(\mathrm{~s}, 1 \mathrm{H}), 7.02(\mathrm{~d}$, $J=8.4 \mathrm{~Hz}, 2 \mathrm{H}), 6.99(\mathrm{~d}, J=8.4 \mathrm{~Hz}, 2 \mathrm{H}), 6.83(\mathrm{~d}, J=8.4 \mathrm{~Hz}$, $2 \mathrm{H}), 3.76(\mathrm{~s}, 3 \mathrm{H}) .{ }^{13} \mathrm{C}$ NMR $\left(100 \mathrm{MHz}, \mathrm{CDCl}_{3}\right) \delta 159.4$, $139.4,133.4,132.1,129.3,129.2,128.8,128.7,128.0$, 127.0, 114.7, 55.5. $\mathrm{C}_{16} \mathrm{H}_{13} \mathrm{ClN}_{2} \mathrm{O}$ (284.74): Calcd C 67.49, H 4.60, N 9.84; Found C 67.28, H 4.37, N 10.08 .

5-(3,5-bis ( trifluoromethyl) phenyl)-1-(4-methoxyphenyl)-1H-imidazole (6) $75 \%$ yield. ${ }^{1} \mathrm{H}$ NMR $\left(400 \mathrm{MHz}, \mathrm{CDCl}_{3}\right) \delta 7.62(\mathrm{~s}, 1 \mathrm{H}), 7.62$ (s, 1H), $7.46(\mathrm{~s}, 2 \mathrm{H}), 7.35(\mathrm{~s}, 1 \mathrm{H}), 7.05(\mathrm{~d}, J=8.4 \mathrm{~Hz}, 2 \mathrm{H})$, 
$6.88(\mathrm{~d}, J=8.4 \mathrm{~Hz}, 2 \mathrm{H}), 3.76(\mathrm{~s}, 3 \mathrm{H}) \cdot{ }^{13} \mathrm{C}$ NMR $(100 \mathrm{MHz}$, $\left.\mathrm{CDCl}_{3}\right) \delta 160.0,140.4,131.7$ (q, $\left.J=34.0 \mathrm{~Hz}\right), 130.4,130.1$, 128.4, $127.3(\mathrm{~m}), 127.1,123.0(\mathrm{q}, J=272.7 \mathrm{~Hz}), 120.6(\mathrm{~m})$, 115.0, 55.6. $\mathrm{C}_{18} \mathrm{H}_{12} \mathrm{~F}_{6} \mathrm{~N}_{2} \mathrm{O}$ (386.29): Calcd C 55.97, H 3.13, N 7.25; Found C 55.79, H 3.20, N 7.41.

4-(5-(4-chlorophenyl)-1H-imidazol-1-yl)benzaldehyde (7) $53 \%$ yield. ${ }^{1} \mathrm{H}$ NMR $\left(400 \mathrm{MHz}, \mathrm{CDCl}_{3}\right) \delta 9.97$ (s, $1 \mathrm{H}), 7.87(\mathrm{~d}, J=8.4 \mathrm{~Hz}, 2 \mathrm{H}), 7.70(\mathrm{~s}, 1 \mathrm{H}), 7.27$ (d, $J=8.4 \mathrm{~Hz}, 2 \mathrm{H}), 7.22(\mathrm{~s}, 1 \mathrm{H}), 7.20(\mathrm{~d}, J=8.4 \mathrm{~Hz}, 2 \mathrm{H}), 6.99$ $(\mathrm{d}, J=8.4 \mathrm{~Hz}, 2 \mathrm{H}) .{ }^{13} \mathrm{C}$ NMR $\left(100 \mathrm{MHz}, \mathrm{CDCl}_{3}\right) \delta 190.7$, $141.1,135.6,134.0,131.0,130.0,129.4,129.0,127.4$, 125.7. $\mathrm{C}_{16} \mathrm{H}_{11} \mathrm{ClN}_{2} \mathrm{O}$ (282.72): Calcd $\mathrm{C} 67.97, \mathrm{H} 3.92, \mathrm{~N}$ 9.91; Found C 67.75, H 3.97, N 9.72.

1-methyl-2,5-diphenyl-1H-imidazole (8) $80 \%$ yield. ${ }^{1} \mathrm{H}$ NMR $\left(400 \mathrm{MHz}, \mathrm{CDCl}_{3}\right) \delta 7.63(\mathrm{~d}, J=8.4 \mathrm{~Hz}, 2 \mathrm{H}), 7.45-$ $7.25(\mathrm{~m}, 8 \mathrm{H}), 7.14(\mathrm{~s}, 1 \mathrm{H}), 3.62(\mathrm{~s}, 3 \mathrm{H})$.

1-methyl-2,5-di-p-tolyl-1H-imidazole (9) $81 \%$ yield. ${ }^{1} \mathrm{H}$ NMR $\left(400 \mathrm{MHz}, \mathrm{CDCl}_{3}\right) \delta 7.52(\mathrm{~d}, J=8.4 \mathrm{~Hz}, 2 \mathrm{H}), 7.27$ (d, $J=8.4 \mathrm{~Hz}, 4 \mathrm{H}), 7.23-7.17(\mathrm{~m}, 4 \mathrm{H}), 7.10(\mathrm{~s}, 1 \mathrm{H}), 3.58$ (s, 3H), $2.34(\mathrm{~s}, 6 \mathrm{H}) .{ }^{13} \mathrm{C}$ NMR $\left(100 \mathrm{MHz}, \mathrm{CDCl}_{3}\right) \delta 148.9$, 139.0, 138.0, 135.3, 129.5, 129.3, 128.8, 128.7, 127.4, 127.1, 126.2, 33.8, 21.4, 21.3. $\mathrm{C}_{18} \mathrm{H}_{18} \mathrm{~N}_{2}$ (262.35): Calcd C 82.41, H 6.92; Found C 82.50, H 6.98.

1-methyl-2,5-di-m-tolyl-1H-imidazole (10) $79 \%$ yield. ${ }^{1} \mathrm{H}$ NMR $\left(400 \mathrm{MHz}, \mathrm{CDCl}_{3}\right) \delta 7.50(\mathrm{~s}, 1 \mathrm{H}), 7.39(\mathrm{~d}$, $J=8.4 \mathrm{~Hz}, 1 \mathrm{H}), 7.30(\mathrm{~d}, J=8.4 \mathrm{~Hz}, 1 \mathrm{H}), 7.26(\mathrm{~d}, J=8.4 \mathrm{~Hz}$, $1 \mathrm{H}), 7.22-7.10(\mathrm{~m}, 5 \mathrm{H}), 3.59(\mathrm{~s}, 3 \mathrm{H}), 2.35(\mathrm{~s}, 3 \mathrm{H}), 2.34(\mathrm{~s}$, $3 \mathrm{H}) .{ }^{13} \mathrm{C}$ NMR $\left(100 \mathrm{MHz}, \mathrm{CDCl}_{3}\right) \delta 174.1,149.1,138.5$, $138.4,135.5,130.3,130.0,129.7,129.5,128.8,128.7$, 128.4, 126.7, 125.8, 125.7, 33.8, 21.5, 21.4. $\mathrm{C}_{18} \mathrm{H}_{18} \mathrm{~N}_{2}$ (262.35): Calcd C 82.41, H 6.92; Found C 82.27, H 6.90.

1-methyl-2,5-di-o-tolyl-1H-imidazole (11) $54 \%$ yield. ${ }^{1} \mathrm{H}$ NMR (400 MHz, CDCl $) \delta 7.38-7.15(\mathrm{~m}, 8 \mathrm{H}), 7.03$ (s, 1H), 3.11 (s, 3H), 2.21 (s, 3H), $2.18(\mathrm{~s}, 3 \mathrm{H}) .{ }^{13} \mathrm{C}$ NMR $\left(100 \mathrm{MHz}, \mathrm{CDCl}_{3}\right) \delta 147.7,138.2,132.4,131.3,130.6$, $130.5,130.4,130.3,129.7,129.4,129.0,126.7,125.9$, 125.8, 31.5, 20.0, 19.7. $\mathrm{C}_{18} \mathrm{H}_{18} \mathrm{~N}_{2}$ (262.35): Calcd C 82.41, H 6.92; Found C 82.50, H 6.98.

4,4'-(1-methyl-1H-imidazole-2,5-diyle)benzonitrile (12) $60 \%$ yield. ${ }^{1} \mathrm{H}$ NMR $\left(400 \mathrm{MHz}, \mathrm{CDCl}_{3}\right) \delta 7.83(\mathrm{~d}$, $J=8.4 \mathrm{~Hz}, 2 \mathrm{H}), 7.73(\mathrm{~d}, J=8.4 \mathrm{~Hz}, 4 \mathrm{H}), 7.55(\mathrm{~d}, J=8.4 \mathrm{~Hz}$, 2H), 7.28 (s, $1 \mathrm{H}), 3.70$ (s, 3H). ${ }^{13} \mathrm{C}$ NMR $(100 \mathrm{MHz}$, $\left.\mathrm{CDCl}_{3}\right) \delta 148.6,134.9,134.1,133.9,132.8,132.6,129.5$, $129.3,128.8,118.4,118.3,112.9,112.0,34.4 . \mathrm{C}_{18} \mathrm{H}_{12} \mathrm{~N}_{4}$ (284.31): Calcd C 76.04, H 4.25; Found C 76.18, H 4.08.
3,3'-(1-methyl-1H-imidazole-2,5-diyle)benzonitrile (13) $60 \%$ yield. ${ }^{1} \mathrm{H}$ NMR $\left(400 \mathrm{MHz}, \mathrm{CDCl}_{3}\right) \delta 7.99$ (s, $1 \mathrm{H}), 7.98(\mathrm{~d}, J=8.4 \mathrm{~Hz}, 1 \mathrm{H}), 7.75-7.65(\mathrm{~m}, 4 \mathrm{H}), 7.61$ $(\mathrm{d}, J=8.4 \mathrm{~Hz}, 1 \mathrm{H}), 7.57(\mathrm{~d}, J=8.4 \mathrm{~Hz}, 1 \mathrm{H}), 7.23(\mathrm{~s}, 1 \mathrm{H})$, $3.68(\mathrm{~s}, 3 \mathrm{H}) .{ }^{13} \mathrm{C}$ NMR $\left(100 \mathrm{MHz}, \mathrm{CDCl}_{3}\right) \delta 146.8,134.1$, $133.4,133.2,133.1,132.4,132.3,132.1,130.2,130.1$, 130.0, 118.0, 117.9, 113.6, 113.3, 34.2. $\mathrm{C}_{18} \mathrm{H}_{12} \mathrm{~N}_{4}$ (284.31): Calcd C 76.04, H 4.25; Found C 76.29, H 4.22.

2,5-bis(4-fluorophényl)-1-methyl-1H-imidazole (14) $78 \%$ yield. ${ }^{1} \mathrm{H}$ NMR $\left(400 \mathrm{MHz}, \mathrm{CDCl}_{3}\right) \delta 7.65-$ $7.55(\mathrm{~m}, 2 \mathrm{H}), 7.40-7.30(\mathrm{~m}, 2 \mathrm{H}), 7.15-7.00(\mathrm{~m}, 5 \mathrm{H})$, $3.57(\mathrm{~s}, 3 \mathrm{H}) .{ }^{13} \mathrm{C}$ NMR $\left(100 \mathrm{MHz}, \mathrm{CDCl}_{3}\right) \delta 163.1(\mathrm{~d}$, $J=249.0 \mathrm{~Hz}), 162.7(\mathrm{~d}, J=249.0 \mathrm{~Hz}), 148.4,134.4,130.7$ $(\mathrm{d}, J=8.3 \mathrm{~Hz}), 130.5(\mathrm{~d}, J=8.3 \mathrm{~Hz}), 127.4,126.9$ (d, $J=3.3 \mathrm{~Hz}), 126.2(\mathrm{~d}, J=3.3 \mathrm{~Hz}), 115.9(\mathrm{~d}, J=17.3 \mathrm{~Hz})$, 115.7 (d, J=17.3 Hz), 33.6. $\mathrm{C}_{16} \mathrm{H}_{12} \mathrm{~F}_{2} \mathrm{~N}_{2}$ (270.28): Calcd C 71.10, H 4.48; Found C 71.02, H 4.34.

2,5-bis(2-fluorophenyl)-1-methyl-1H-imidazole (15) $59 \%$ yield. ${ }^{1} \mathrm{H}$ NMR $\left(400 \mathrm{MHz}, \mathrm{CDCl}_{3}\right) \delta 7.56(\mathrm{t}$, $J=8.0 \mathrm{~Hz}, 1 \mathrm{H}), 7.42-7.30(\mathrm{~m}, 3 \mathrm{H}), 7.25-7.08(\mathrm{~m}, 5 \mathrm{H})$, $3.42(\mathrm{~s}, 3 \mathrm{H}) .{ }^{13} \mathrm{C}$ NMR $\left(100 \mathrm{MHz}, \mathrm{CDCl}_{3}\right) \delta 160.0(\mathrm{dd}$, $J=249.2,7.4 \mathrm{~Hz}), 144.7,132.4(\mathrm{~d}, J=2.7 \mathrm{~Hz}), 131.9(\mathrm{~d}$, $J=2.7 \mathrm{~Hz}), 131.2(\mathrm{~d}, J=8.1 \mathrm{~Hz}), 130.4(\mathrm{~d}, J=8.1 \mathrm{~Hz})$, 129.3, 124.6 (d, $J=3.4 \mathrm{~Hz}), 124.4(\mathrm{~d}, J=3.4 \mathrm{~Hz}), 119.1$ (d, $J=14.9 \mathrm{~Hz}), 118.1(\mathrm{~d}, J=14.9 \mathrm{~Hz}), 116.1(\mathrm{~d}, J=13.2 \mathrm{~Hz})$, 115.9 (d, $J=13.2 \mathrm{~Hz}$ ), 32.4. $\mathrm{C}_{16} \mathrm{H}_{12} \mathrm{~F}_{2} \mathrm{~N}_{2}$ (270.28): Calcd C 71.10, H 4.48; Found C 71.27, H 4.55.

1-méthyl-2,5-bis(3-(trifluoromethyl)phenyl)-1H-imidazole (16) $59 \%$ yield. ${ }^{1} \mathrm{H}$ NMR $\left(400 \mathrm{MHz}, \mathrm{CDCl}_{3}\right) \delta$ $7.92(\mathrm{~s}, 1 \mathrm{H}), 7.83(\mathrm{~d}, J=8.4 \mathrm{~Hz}, 1 \mathrm{H}), 7.65-7.50(\mathrm{~m}, 6 \mathrm{H})$, 7.21 (s, 1H), 3.63 (s, 3H) ${ }^{13} \mathrm{C}$ NMR $\left(100 \mathrm{MHz}, \mathrm{CDCl}_{3}\right) \delta$ $148.5,134.5,131.9,131.8,131.5$ (q, $J=20.4 \mathrm{~Hz}), 131.4$, 131.1 (q, $J=20.4 \mathrm{~Hz}), 130.7,129.4,129.2,128.6,125.7$, $(\mathrm{q}, J=3.7 \mathrm{~Hz}), 125.6(\mathrm{q}, J=3.7 \mathrm{~Hz}), 125.3(\mathrm{q}, J=3.7 \mathrm{~Hz})$, 124.8 (q, J=3.7 Hz), 122.5, 33.8. $\mathrm{C}_{18} \mathrm{H}_{12} \mathrm{~F}_{6} \mathrm{~N}_{2}$ (370.29): Calcd C 58.38, H 3.27; Found C 58.47, H 3.45.

2,5-bis(4-chlorophenyl)-1-methyl-1H-imidazole (17) $70 \%$ yield. ${ }^{1} \mathrm{H}$ NMR $\left(400 \mathrm{MHz}, \mathrm{CDCl}_{3}\right) \delta 7.56(\mathrm{~d}$, $J=8.4 \mathrm{~Hz}, 2 \mathrm{H}), 7.39(\mathrm{~d}, J=8.4 \mathrm{~Hz}, 2 \mathrm{H}), 7.37(\mathrm{~d}, J=8.4 \mathrm{~Hz}$, $2 \mathrm{H}), 7.30(\mathrm{~d}, J=8.4 \mathrm{~Hz}, 2 \mathrm{H}), 7.12(\mathrm{~s}, 1 \mathrm{H}), 3.58(\mathrm{~s}, 3 \mathrm{H}) .{ }^{13} \mathrm{C}$ NMR $\left(100 \mathrm{MHz}, \mathrm{CDCl}_{3}\right) \delta 148.2,135.3,134.6,134.4$, $130.1,129.9,129.2,129.0,128.5,128.2,127.1,33.9$. $\mathrm{C}_{16} \mathrm{H}_{12} \mathrm{Cl}_{2} \mathrm{~N}_{2}$ (303.19): Calcd C 63.38, H 3.99; Found C 63.55, H 4.09.

1-methyl-2,5-bis(4-nitrophenyl)-1H-imidazole (18) 32\% yield. ${ }^{1} \mathrm{H}$ NMR $\left(400 \mathrm{MHz}, \mathrm{CDCl}_{3}\right) \delta 8.39(\mathrm{~d}, J=8.4 \mathrm{~Hz}$, $2 \mathrm{H}), 8.36(\mathrm{~d}, J=8.4 \mathrm{~Hz}, 2 \mathrm{H}), 8.07(\mathrm{~d}, J=8.4 \mathrm{~Hz}, 2 \mathrm{H}), 7.89$ $(\mathrm{d}, J=8.4 \mathrm{~Hz}, 2 \mathrm{H}), 7.55$ (s, 1H), $3.83(\mathrm{~s}, 3 \mathrm{H}) .{ }^{13} \mathrm{C}$ NMR 
$\left(100 \mathrm{MHz}, \mathrm{CDCl}_{3}\right) \delta 148.7,147.6,147.0,136.8,136.4$, 135.1, 130.9, 130.0, 129.3, 124.6, 124.3, 35.0. $\mathrm{C}_{16} \mathrm{H}_{12} \mathrm{~N}_{4} \mathrm{O}_{4}$ (324.29): Calcd C 59.26, H 3.73; Found C 59.04, H 3.49.

1-methyl-2,5-bis(3-nitrophenyl)-1H-imidazole (19) 62\% yield. ${ }^{1} \mathrm{H}$ NMR $\left(400 \mathrm{MHz}, \mathrm{CDCl}_{3}\right) \delta 8.52(\mathrm{~s}, 1 \mathrm{H}), 8.35-$ $8.15(\mathrm{~m}, 3 \mathrm{H}), 8.05(\mathrm{~d}, J=8.4 \mathrm{~Hz}, 1 \mathrm{H}), 7.74(\mathrm{~d}, J=8.4 \mathrm{~Hz}$, $1 \mathrm{H}), 7.65(\mathrm{~d}, J=8.4 \mathrm{~Hz}, 1 \mathrm{H}), 7.62(\mathrm{~d}, J=8.4 \mathrm{~Hz}, 1 \mathrm{H}), 7.25$ (s, $1 \mathrm{H}), 3.72(\mathrm{~s}, 3 \mathrm{H}) \cdot{ }^{13} \mathrm{C}$ NMR $\left(100 \mathrm{MHz}, \mathrm{CDCl}_{3}\right) \delta 148.6$, $148.4,147.9,134.7,134.3,134.0,131.9,131.3,130.1$, 130.0, 129.4, 123.8, 123.4, 123.1, 123.0, 34.0. $\mathrm{C}_{16} \mathrm{H}_{12} \mathrm{~N}_{4} \mathrm{O}_{4}$ (324.29): Calcd C 59.26, H 3.73; Found C 59.40, H 3.61 .

2,5-bis(4-methoxyphenyl)-1-methyl-1H-imidazole (20) $78 \%$ yield. ${ }^{1} \mathrm{H}$ NMR $\left(400 \mathrm{MHz}, \mathrm{CDCl}_{3}\right) \delta 7.55(\mathrm{~d}$, $J=8.4 \mathrm{~Hz}, 2 \mathrm{H}), 7.30(\mathrm{~d}, J=8.4 \mathrm{~Hz}, 2 \mathrm{H}), 7.04(\mathrm{~s}, 1 \mathrm{H}), 6.93$ $(\mathrm{d}, J=8.4 \mathrm{~Hz}, 2 \mathrm{H}), 6.92(\mathrm{~d}, J=8.4 \mathrm{~Hz}, 2 \mathrm{H}), 3.80(\mathrm{~s}, 3 \mathrm{H})$, $3.79(\mathrm{~s}, 3 \mathrm{H}), 3.55(\mathrm{~s}, 3 \mathrm{H})$.

4,4'-(1-methyl-1H-imidazole-2,5-diyle)dibenzaldehyde (21) $62 \%$ yield. ${ }^{1} \mathrm{H}$ NMR (400 MHz, $\left.\mathrm{CDCl}_{3}\right) \delta 10.03$ (s, 1H), 10.01 (s, 1H), $7.96(\mathrm{~d}, J=8.4 \mathrm{~Hz}, 2 \mathrm{H}), 7.93$ (d, $J=8.4 \mathrm{~Hz}, 2 \mathrm{H}), 7.85(\mathrm{~d}, J=8.4 \mathrm{~Hz}, 2 \mathrm{H}), 7.59(\mathrm{~d}, J=8.4 \mathrm{~Hz}$, 2H), 7.33 (s, $1 \mathrm{H}), 3.73$ (s, 3H). ${ }^{13} \mathrm{C}$ NMR $(100 \mathrm{MHz}$, $\left.\mathrm{CDCl}_{3}\right) \delta 191.6,191.4,149.3,136.2,135.9,135.6,131.5$, 130.3, 130.0, 129.8, 129.2, 128.7, 127.5, 34.4. $\mathrm{C}_{18} \mathrm{H}_{14} \mathrm{~N}_{2} \mathrm{O}_{2}$ (290.32): Calcd C 74.47, H 4.86; Found C 74.55, H 4.99.

1,1'-((1-methyl-1H-imidazole-2,5-diyl)bis(4,1-phenylene)) bis(propane-1-one) (22) $65 \%$ yield. ${ }^{1} \mathrm{H}$ NMR $(400 \mathrm{MHz}$, $\left.\mathrm{CDCl}_{3}\right) \delta 8.02(\mathrm{~d}, J=8.4 \mathrm{~Hz}, 2 \mathrm{H}), 7.98(\mathrm{~d}, J=8.4 \mathrm{~Hz}$, $2 \mathrm{H}), 7.75(\mathrm{~d}, J=8.4 \mathrm{~Hz}, 2 \mathrm{H}), 7.50(\mathrm{~d}, J=8.4 \mathrm{~Hz}, 2 \mathrm{H})$, $7.27(\mathrm{~s}, 1 \mathrm{H}), 3.71(\mathrm{~s}, 3 \mathrm{H}), 2.95(\mathrm{q}, J=7.5 \mathrm{~Hz}, 4 \mathrm{H}), 1.15$ $(\mathrm{t}, J=7.5 \mathrm{~Hz}, 6 \mathrm{H}) .{ }^{13} \mathrm{C}$ NMR $\left(100 \mathrm{MHz}, \mathrm{CDCl}_{3}\right) \delta 200.1$, $200.0,149.0,136.9,136.2,135.3,134.1,133.9,128.9$, 128.6, 128.4, 128.3, 34.4, 32.0, 31.9, 8.3, 8.2. $\mathrm{C}_{22} \mathrm{H}_{22} \mathrm{~N}_{2} \mathrm{O}_{2}$ (346.42): Calcd C 76.28, H 6.40; Found C 76.08, H 6.21.

1,1'-((1-methyl-1H-imidazole-2,5-diyl)bis(4,1-phenylene)) bis(propane-1-one) (23) $65 \%$ yield. ${ }^{1} \mathrm{H}$ NMR (400 MHz, $\left.\mathrm{CDCl}_{3}\right) \delta 8.02(\mathrm{~d}, J=8.4 \mathrm{~Hz}, 2 \mathrm{H}), 7.98(\mathrm{~d}, J=8.4 \mathrm{~Hz}$, $2 \mathrm{H}), 7.75(\mathrm{~d}, J=8.4 \mathrm{~Hz}, 2 \mathrm{H}), 7.50(\mathrm{~d}, J=8.4 \mathrm{~Hz}, 2 \mathrm{H})$, $7.27(\mathrm{~s}, 1 \mathrm{H}), 3.71(\mathrm{~s}, 3 \mathrm{H}), 2.95(\mathrm{q}, J=7.5 \mathrm{~Hz}, 4 \mathrm{H}), 1.15$ $(\mathrm{t}, J=7.5 \mathrm{~Hz}, 6 \mathrm{H}) .{ }^{13} \mathrm{C}$ NMR $\left(100 \mathrm{MHz}, \mathrm{CDCl}_{3}\right) \delta 200.1$, $200.0,149.0,136.9,136.2,135.3,134.1,133.9,128.9$, 128.6, 128.4, 128.3, 34.4, 32.0, 31.9, 8.3, 8.2. $\mathrm{C}_{22} \mathrm{H}_{22} \mathrm{~N}_{2} \mathrm{O}_{2}$ (346.42): Calcd C 76.28, H 6.40.; Found C 76.08, H 6.21.

1-benzyl-5-(4-chlorophenyl)-1H-imidazole (24) $18 \%$ yield. ${ }^{1} \mathrm{H}$ NMR $\left(400 \mathrm{MHz}, \mathrm{CDCl}_{3}\right) \delta 7.51(\mathrm{~s}, 1 \mathrm{H}), 7.28-$ $7.15(\mathrm{~m}, 5 \mathrm{H}), 7.12(\mathrm{~d}, J=8.4 \mathrm{~Hz}, 2 \mathrm{H}), 7.06(\mathrm{~s}, 1 \mathrm{H}), 6.92(\mathrm{~d}$, $J=8.4 \mathrm{~Hz}, 2 \mathrm{H}), 5.06(\mathrm{~s}, 2 \mathrm{H}) .{ }^{13} \mathrm{C} \mathrm{NMR}\left(100 \mathrm{MHz}, \mathrm{CDCl}_{3}\right)$ $\delta 139.0,136.5,134.2,132.3,130.1,129.0,128.9,128.6$, 128.2, 128.1,126.5, 48.8 .

1-butyl-2,5-di-p-tolyl-1H-imidazole (25) $25 \%$ yield. ${ }^{1} \mathrm{H}$ NMR $\left(400 \mathrm{MHz}, \mathrm{CDCl}_{3}\right) \delta 7.46(\mathrm{~d}, J=8.4 \mathrm{~Hz}, 2 \mathrm{H}), 7.25$ (d, $J=8.4 \mathrm{~Hz}, 2 \mathrm{H}), 7.23-7.17(\mathrm{~m}, 4 \mathrm{H}), 7.02(\mathrm{~s}, 1 \mathrm{H}), 3.99$ $(\mathrm{t}, J=7.5 \mathrm{~Hz}, 2 \mathrm{H}), 2.34(\mathrm{~s}, 6 \mathrm{H}), 1.26-1.15(\mathrm{~m}, 2 \mathrm{H}), 0.90$ $0.83(\mathrm{~m}, 2 \mathrm{H}), 0.54(\mathrm{t}, J=7.5 \mathrm{~Hz}, 3 \mathrm{H}) .{ }^{13} \mathrm{C}$ NMR $(100 \mathrm{MHz}$, $\left.\mathrm{CDCl}_{3}\right) \delta 149.1,138.5,137.8,134.3,129.4,129.2,128.8$, 128.7, 128.0, 127.7, 44.7, 32.3, 21.4, 21.3, 19.3, 13.3. $\mathrm{C}_{21} \mathrm{H}_{24} \mathrm{~N}_{2}$ (304.43): Calcd C 82.85, H 7.95; Found C 82.67, $\mathrm{H} 8.14$.

1-butyl-2,5-bis(4-chlorophenyl)-1H-imidazole (26) 24\% yield. ${ }^{1} \mathrm{H}$ NMR $\left(400 \mathrm{MHz}, \mathrm{CDCl}_{3}\right) \delta 7.48(\mathrm{~d}, J=8.4 \mathrm{~Hz}$, $2 \mathrm{H}), 7.39(\mathrm{~d}, J=8.4 \mathrm{~Hz}, 2 \mathrm{H}), 7.38(\mathrm{~d}, J=8.4 \mathrm{~Hz}, 2 \mathrm{H}), 7.31$ (d, $J=8.4 \mathrm{~Hz}, 2 \mathrm{H}), 7.06(\mathrm{~s}, 1 \mathrm{H}), 3.99(\mathrm{t}, J=7.5 \mathrm{~Hz}, 2 \mathrm{H})$, $1.26-1.15(\mathrm{~m}, 2 \mathrm{H}), 0.90-0.83(\mathrm{~m}, 2 \mathrm{H}), 0.57(\mathrm{t}, J=7.5 \mathrm{~Hz}$, $3 \mathrm{H}) .{ }^{13} \mathrm{C}$ NMR $\left(100 \mathrm{MHz}, \mathrm{CDCl}_{3}\right) \delta 148.3,135.0,134.2$, 133.6, 130.2, 130.1, 129.9, 129.1, 128.9, 128.5, 44.9, 32.4, 19.3, 13.3. $\mathrm{C}_{19} \mathrm{H}_{18} \mathrm{Cl}_{2} \mathrm{~N}_{2}$ (345.27): Calcd C 66.09, H 5.25; Found C 66.14, H 5.08.

2,5-bis(3,5-bis(trifluoromethyl)phenyl)-1-methyl-1H-imidazole (27) $48 \%$ yield. ${ }^{1} \mathrm{H}$ NMR $\left(400 \mathrm{MHz}, \mathrm{CDCl}_{3}\right) \delta$ $8.13(\mathrm{~s}, 2 \mathrm{H}), 7.89(\mathrm{~s}, 1 \mathrm{H}), 7.85(\mathrm{~s}, 3 \mathrm{H}), 7.31(\mathrm{~s}, 1 \mathrm{H}), 3.70$ $(\mathrm{s}, 3 \mathrm{H}) .{ }^{13} \mathrm{C}$ NMR $\left(100 \mathrm{MHz}, \mathrm{CDCl}_{3}\right) \delta 147.6,133.8,132.7$ $(\mathrm{q}, J=26.2 \mathrm{~Hz}), 132.1$ (q, $J=26.2 \mathrm{~Hz}), 131.5,129.6,128.5$, 128.4, 124.3, 122.8 (quint., $J=3.6 \mathrm{~Hz}$ ), 122.1 (quint., $J=3.6 \mathrm{~Hz}), 121.6,118.9$, 33.9. $\mathrm{C}_{20} \mathrm{H}_{10} \mathrm{~F}_{12} \mathrm{~N}_{2}$ (506.29): Calcd C 47.45, H 1.99, N 5.53; Found C 47.40, H 2.09, N 5.36.

1-benzyl-2,5-bis(3,5-bis(trifluoromethyl)phenyl)-1H-imidazole (28) $32 \%$ yield. ${ }^{1} \mathrm{H}$ NMR $\left(400 \mathrm{MHz}, \mathrm{CDCl}_{3}\right) \delta$ $8.01(\mathrm{~s}, 2 \mathrm{H}), 7.81(\mathrm{~s}, 1 \mathrm{H}), 7.76(\mathrm{~s}, 1 \mathrm{H}), 7.67(\mathrm{~s}, 2 \mathrm{H}), 7.38$ (s, $1 \mathrm{H}), 7.28-7.20(\mathrm{~m}, 3 \mathrm{H}), 6.81(\mathrm{~d}, J=8.4 \mathrm{~Hz}, 2 \mathrm{H}), 5.19$ (s, 2H). ${ }^{13} \mathrm{C}$ NMR $\left(100 \mathrm{MHz}, \mathrm{CDCl}_{3}\right) \delta 147.7,135.8$, $133.5,132.4$ (q, $J=34.0 \mathrm{~Hz}), 132.3(\mathrm{q}, J=34.0 \mathrm{~Hz}), 132.2$, 131.6, 130.4, 129.4, 128.7 (m), 128.5, 125.4, 122.9 (q, $J=272.7 \mathrm{~Hz}), 122.8(\mathrm{~m}), 122.7(\mathrm{q}, J=272.7 \mathrm{~Hz}), 122.0$ (m), 49.3. $\mathrm{C}_{26} \mathrm{H}_{14} \mathrm{~F}_{12} \mathrm{~N}_{2}$ (582.38): Calcd C 53.62, H 2.42, N 4.81; Found C 53.60, H 2.54, N 4.88

2,5-bis(3,5-bis(trifluoromethyl)phenyl)-1-butyl-1H-imidazole (29) $35 \%$ yield. ${ }^{1} \mathrm{H}$ NMR $\left(400 \mathrm{MHz}, \mathrm{CDCl}_{3}\right) \delta$ $8.05(\mathrm{~s}, 2 \mathrm{H}), 7.91(\mathrm{~s}, 1 \mathrm{H}), 7.88(\mathrm{~s}, 1 \mathrm{H}), 7.84(\mathrm{~s}, 2 \mathrm{H}), 7.26(\mathrm{~s}$, $1 \mathrm{H}), 4.04(\mathrm{t}, J=7.5 \mathrm{~Hz}, 2 \mathrm{H}), 1.40-1.25(\mathrm{~m}, 2 \mathrm{H}), 1.05-0.90$ (m, 2H), 0.60 (t, $J=7.5 \mathrm{~Hz}, 3 \mathrm{H}) .{ }^{13} \mathrm{C}$ NMR $(100 \mathrm{MHz}$, $\left.\mathrm{CDCl}_{3}\right) \delta 147.3,133.0-131.5(\mathrm{~m}), 130.4,128.8,128.6$, $127.1,124.4,122.8(\mathrm{q}, J=J=3.7 \mathrm{~Hz}), 122.1(\mathrm{q}, J=3.7 \mathrm{~Hz})$, 121.6, 118.9, 45.4, 32.6, 19.2, 13.0. $\mathrm{C}_{23} \mathrm{H}_{16} \mathrm{~F}_{12} \mathrm{~N}_{2}$ (548.37): 
Calcd C 50.38, H 2.94, N 5.11; Found C 50.19, H 2.78, N 4.83 .

4-(2,5-bis(3,5-bis(trifluoromethyl)phenyl)-1H-imidazol-1-yl)benzaldehyde (30) The product was obtained as trace observed by GC/MS analysis of the crude mixture.

2, 5 -bis ( 3, 5-bis (trifluoromethyl) phenyl)-1-(4-methoxyphenyl)-1H-imidazole (31) Low yield. ${ }^{1} \mathrm{H}$ NMR $\left(400 \mathrm{MHz}, \mathrm{CDCl}_{3}\right) \delta 7.79(\mathrm{~s}, 2 \mathrm{H}), 7.70$ (s, 1H), $7.66(\mathrm{~s}, 1 \mathrm{H}), 7.50(\mathrm{~s}, 1 \mathrm{H}), 7.47(\mathrm{~s}, 2 \mathrm{H}), 7.03(\mathrm{~d}$, $J=8.4 \mathrm{~Hz}, 2 \mathrm{H}), 6.92(\mathrm{~d}, J=8.4 \mathrm{~Hz}, 2 \mathrm{H}), 3.78(\mathrm{~s}, 3 \mathrm{H})$.

2,4,5-tris(3,5-bis(trifluoromethyl)phenyl)-1-methyl-1H-imidazole (32) $30 \%$ yield. ${ }^{1} \mathrm{H}$ NMR $(400 \mathrm{MHz}$, $\left.\mathrm{CDCl}_{3}\right) \delta 8.18(\mathrm{~s}, 2 \mathrm{H}), 8.00(\mathrm{~s}, 1 \mathrm{H}), 7.96(\mathrm{~s}, 1 \mathrm{H}), 7.84$ (s, 4H), $7.66(\mathrm{~s}, 1 \mathrm{H}), 3.59(\mathrm{~s}, 3 \mathrm{H}) \cdot{ }^{13} \mathrm{C}$ NMR $(100 \mathrm{MHz}$, $\left.\mathrm{CDCl}_{3}\right) \delta 145.7,136.4,134.0,132.3(\mathrm{q}, J=34.8 \mathrm{~Hz}), 131.5$ (q, $J=34.8 \mathrm{~Hz}), 130.9$ (q, $J=34.8 \mathrm{~Hz}), 130.7,130.6,129.6$ $(\mathrm{m}), 128.6,128.0(\mathrm{~m}), 122.4(\mathrm{~m}), 122.2(\mathrm{~m}), 120.0(\mathrm{~m})$, $121.7(\mathrm{q}, J=270.0 \mathrm{~Hz}), 121.6(\mathrm{q}, J=270.0 \mathrm{~Hz}), 121.5(\mathrm{q}$, $J=270.0 \mathrm{~Hz}$ ), 32.6. $\mathrm{C}_{28} \mathrm{H}_{12} \mathrm{~F}_{18} \mathrm{~N}_{2}$ (718.38): Calcd C 46.81, H 1.68; Found C 46.99, H 1.88.

2,4,5-tris(3,5-bis(trifluoromethyl)phenyl)-1-butyl-1H-imidazole (33) $33 \%$ yield. ${ }^{1} \mathrm{H}$ NMR $\left(400 \mathrm{MHz}, \mathrm{CDCl}_{3}\right) \delta$ $8.11(\mathrm{~s}, 2 \mathrm{H}), 8.02(\mathrm{~s}, 1 \mathrm{H}), 7.97$ (s, 1H), 7.85 (s, 2H), 7.78 $(\mathrm{s}, 2 \mathrm{H}), 7.64(\mathrm{~s}, 1 \mathrm{H}), 3.89(\mathrm{t}, J=7.5 \mathrm{~Hz}, 2 \mathrm{H}), 1.40-1.25(\mathrm{~m}$, $2 \mathrm{H}), 1.05-0.90(\mathrm{~m}, 2 \mathrm{H}), 0.60(\mathrm{t}, J=7.5 \mathrm{~Hz}, 3 \mathrm{H})$.

1-benzyl-2,4,5-tris(3,5-bis(trifluoromethyl) phenyl)-1H-imidazole (34) $34 \%$ yield. ${ }^{1} \mathrm{H} \quad \mathrm{NMR}$ $\left(400 \mathrm{MHz}, \mathrm{CDCl}_{3}\right) \delta 8.00(\mathrm{~s}, 2 \mathrm{H}), 7.89(\mathrm{~s}, 1 \mathrm{H}), 7.87(\mathrm{~s}$, $1 \mathrm{H}), 7.84(\mathrm{~s}, 2 \mathrm{H}), 7.64(\mathrm{~s}, 1 \mathrm{H}), 7.59(\mathrm{~s}, 2 \mathrm{H}), 7.25-7.20(\mathrm{~m}$, $3 \mathrm{H}), 6.736 .81(\mathrm{~d}, J=8.4 \mathrm{~Hz}, 2 \mathrm{H}), 5.08(\mathrm{~s}, 2 \mathrm{H}) .{ }^{13} \mathrm{C}$ NMR $\left(100 \mathrm{MHz}, \mathrm{CDCl}_{3}\right) \delta 146.9,137.7,135.2,134.9,133.1$ (q, $J=34.0 \mathrm{~Hz}), 132.5(\mathrm{q}, J=34.0 \mathrm{~Hz}), 131.1,131.7,131.6$, $130.8(\mathrm{~m}), 129.4,129.0(\mathrm{~m}), 128.7,126.5(\mathrm{~m}), 125.6,123.3$ (m), $122.9(\mathrm{q}, J=272.7 \mathrm{~Hz}), 122.7(\mathrm{q}, J=272.7 \mathrm{~Hz}), 122.5$ (q, $J=272.7 \mathrm{~Hz}), 120.9(\mathrm{~m}), 49.4$.

\section{Bacterial strains and determination of the antibacterial activity}

The antibacterial activity has been determined using the disc diffusion assay as previously described [36]. The measurements of inhibition zones were carried out three times for each drug including the antibiotic streptomycin as a positive control. Five bacterial strains were used in this study: Escherichia coli (DH5 $\alpha$ ), Citrobacter freundii, Salmonella braenderup, Staphylococcus aureus, and Listeria monocytogenes. The last four strains were provided from the Pasteur Institute of Casablanca Morocco.
Fungal strains and determination of the antifungal activity The compounds were evaluated for their antifungal activity using liquid cell culture against Saccharomyces cerevisiae (BY4741) and two Candida species: Candida albicans (SC5314) and Candida krusei (ATCC6258). Growth rate of yeast cells in liquid culture was monitored by measuring the absorbance of the cells at $600 \mathrm{~nm}\left(\mathrm{OD}_{600}\right)$ using a V-1200 spectrophotometer (Shanghai Mapada Instruments CO., LTD). The antifungal activity of a compound was evaluated as follows: Cells were grown overnight in yeast peptone dextrose medium (YPD) at $30{ }^{\circ} \mathrm{C}$ in a shaking incubator. Cells were then diluted to an $\mathrm{OD}_{600}$ of $\sim 0.08$ and allowed to grow until the $\mathrm{OD}_{600}$ reached $\sim 0.14$, to ensure that the cells were in logarithmic phase. Compound was then added and the $\mathrm{OD}_{600}$ was measured after $24 \mathrm{~h}$ of cell growth. The relative growth of yeast cells in the presence of a compound was then obtained by calculating the ratio of the $\mathrm{OD}_{600}$ determined for the treated cells to the $\mathrm{OD}_{600}$ of the untreated cells. All experiments were repeated at least twice and means were calculated.

\section{Determination of the $\mathrm{IC}_{\mathbf{5 0}}$}

$\mathrm{IC}_{50}$ is the concentration at which growth is inhibited by $50 \%$ in the presence of the compound. $\mathrm{IC}_{50} \mathrm{~s}$ were determined as previously described [37].

\section{Statistical analysis}

Statistical analyses were performed using one-way ANOVA Test in SPSS software version 21.0. The results were statistically considered significant when $p$ value $<0.05$.

\section{In silico ADME and toxicity predictions}

In silico screening for prediction of the ADME properties (absorption, distribution, metabolism, excretion) of the studied compounds was performed with Molinspiration, a web-based software, (http://www.molinspira tion.com), while screening for toxicity risks (mutagenicity, tumorogenicity, irritation, reproduction) was carried out with DataWarroir software [26, 38, 39].

\section{Molecular docking studies}

The chemical structures of the studied molecules were sketched using ACD/ChemSketch, then optimized by the DFT/B3LYP method with 6-31G(d,p) basis sets using Gaussian 09 software [40].

The crystal structure of lanosterol $14 \alpha$-demethylase from $S$. cerevisiae co-crystallized with the azole antifungal ligand Fluconazole (PDB: 4wmz) was obtained from the Protein Data Bank (http://www.pdb.org) and used as a target in docking studies. The docking studies 
were carried out using SwissDock web server which is based on the docking software EADock DSS [5, 41]. The analysis and the visualization of the docking results were performed using the UCSF Chimera molecular viewer [42].

\section{Additional file}

Additional file 1. Supporting document showing the ${ }^{1} \mathrm{H}$ and ${ }^{13} \mathrm{C}$ NMR spectra of each compound studied in this paper.

\section{Abbreviations}

$\mathrm{IC}_{50}$ : the concentration at which growth is inhibited by 50\%; SAR: struc ture-activity relationship analysis; ADME: absorption, distribution, metabolism excretion properties; Tox: toxicity risks; CYP51: fungal lanosterol 14a-demethylase.

\section{Acknowledgements}

We are grateful to Prof. A. Azzouzi and all the administrative staff of the Faculty of Medicine and Pharmacy of Oujda for their valuable support and encouragement throughout the entire work. We thank Prof. A. Sellam (Université Laval, Canada) and Prof. S. Mentak for proof-reading the manuscript. We also thank the MESRSFC (Ministère de l'Enseignement Supérieur, de la Recherche Scientifique et de la Formation des Cadres) and the CNRST (Centre National pour la Recherche Scientifique et Technique) for their support.

\section{Authors' contributions}

$\mathrm{BB}, \mathrm{FA}, \mathrm{RT}$ and $\mathrm{MB}$ conceived and designed the study. AT synthesized all compounds. PHD, HD and RT supervised the synthesis of all compounds. $\mathrm{BB}$ carried out the all the biological tests. BB, FA, and MB carried out the SAR, ADME-Tox and docking analysis. BB, FA, and MB wrote the manuscript. All authors read and approved the final manuscript.

\section{Funding}

No funding was received for this work.

\section{Availability of data and materials}

All data generated or analyzed during this study are included in this published article.

\section{Competing interests}

The authors declare that they have no competing interests.

\section{Author details}

${ }^{1}$ Genetics Unit, Faculty of Medicine and Pharmacy of Oujda, University Mohammed Premier, Oujda, Morocco. ${ }^{2}$ Laboratory of Applied Chemistry \& Environment, Faculty of Sciences, University Premier, Oujda, Morocco Mohamed Premier, Oujda, Morocco. ${ }^{3}$ Institut des Sciences Chimiques de Rennes, UMR 6226, CNRS, Université de Rennes, Rennes, France.

Received: 14 February 2019 Accepted: 31 July 2019

Published online: 06 August 2019

\section{References}

1. The top 10 causes of death. World Health Organization. 2018. http:// www.who.int/en/news-room/fact-sheets/detail/the-top-10-causes-ofdeath. Accessed 13 Feb 2019

2. Smith KF, Goldberg M, Rosenthal S, Carlson L, Chen J, Chen C et al (2014) Global rise in human infectious disease outbreaks. J Royal Soc Interface. 11(101):20140950

3. Davies J, Davies D (2010) Origins and evolution of antibiotic resistance. Microbiol Mol Biol Rev 74(3):417-433
4. Gandra S, Barter D, Laxminarayan R (2014) Economic burden of antibiotic resistance: how much do we really know? Clin Microbiol Infect 20(10):973-980

5. Abrigach F, Rokni Y, Takfaoui A, Khoutoul M, Doucet $H$, Asehraou A, Touzani R (2018) In vitro screening, homology modeling and molecular docking studies of some pyrazole and imidazole derivatives. Biomed Pharmacother 103:653-661

6. Vandeputte P, Ferrari S, Coste AT (2012) Antifungal resistance and new strategies to control fungal infections. Int J Microbiol. 2012:713687

7. Pfaller MA (2012) Antifungal drug resistance: mechanisms, epidemiology, and consequences for treatment. Am J Med 125(1):S3-S13

8. Sanglard D (2016) Emerging threats in antifungal-resistant fungal pathogens. Front Med. 3:11

9. Perlin DS, Rautemaa-Richardson R, Alastruey-Izquierdo A (2017) The global problem of antifungal resistance: prevalence, mechanisms, and management. Lancet Infect Dis. 17:e383-e392

10. Limper AH, Adenis A, Le T, Harrison TS (2017) Fungal infections in HIV/ AIDS. Lancet Infect Dis. 17:e334-e343

11. Saini MS, Kumar A, Dwivedi J, Singh R (2013) A review: biological significances of heterocyclic compounds. Int J Pharm Sci Res. 4(3):66-77

12. Thomas R, Hossain M, Mary YS, Resmi K, Armaković S, Armaković SJ, Nanda AK, Ranjan VK, Vijayakumar G, Van Alsenoy C (2018) Spectroscopic analysis and molecular docking of imidazole derivatives and investigation of its reactive properties by DFT and molecular dynamics simulations. J Mol Struct 1158:156-175

13. Smitha M, Mary YS, Hossain M, Resmi K, Armaković S, Armaković SJ, Pavithran R, Nanda AK, Van Alsenoy C (2018) Two novel imidazole derivatives_combined experimental and computational study. J Mol Struct 1173:221-239

14. Gupta V, Kant V (2013) A review on biological activity of imidazole and thiazole moieties and their derivatives. Sci Int. 1(7):253-260

15. Adib M, Ansari S, Feizi S, Damavandi JA, Mirzaei P (2009) A one-pot, four-component synthesis of $\mathrm{N}$-substituted 2, 4-diarylimidazoles. Synlett 2009(20):3263-3266

16. Husain A, Drabu S, Kumar N, Alam M, Bawa S (2013) Synthesis and biological evaluation of di-and tri-substituted imidazoles as safer antiinflammatory-antifungal agents. J Pharm Bioall Sci. 5(2):154

17. Sharma A, Kumar V, Kharb R, Kumar S, Chander Sharma P, Pal Pathak D (2016) Imidazole derivatives as potential therapeutic agents. Curr Pharm Des 22(21):3265-3301

18. Shalmali N, Ali MR, Bawa S (2018) Imidazole: an essential edifice for the identification of new lead compounds and drug development. Mini-Rev Med Chem 18(2):142-163

19. Lackner TE, Clissold SP (1989) Bifonazole A review of its antimicrobial activity and therapeutic use in superficial mycoses. Drugs. 38(2):204-225

20. Como JA, Dismukes WE (1994) Oral azole drugs as systemic antifungal therapy. N Engl J Med 330(4):263-272

21. Leitsch D (2017) A review on metronidazole: an old warhorse in antimicrobial chemotherapy. Parasitol. 23:1-12

22. Pattichis K, Louca LL (1995) Histamine, histamine H2-receptor antagonists, gastric acid secretion and ulcers: an overview. Drug Metabol Drug Interact. 12(1):1-36

23. Xu F-Y, Yang B, Shi D, Li H, Zou Z, Shi X-Y (2012) Antihypertensive effects and safety of eprosartan: a meta-analysis of randomized controlled trials. Eur J Clin Pharmacol 68(2):195-205

24. Takfaoui A, Zhao L, Touzani R, Soulé J-F, Dixneuf PH, Doucet H (2014) One pot Pd (OAc) 2-catalysed 2, 5-diarylation of imidazoles derivatives. Tetrahedron 70(44):8316-8323

25. Alqahtani S (2017) In silico ADME-Tox modeling: progress and prospects. Expert Opin Drug Metab Toxicol. 13(11):1147-1158

26. Lipinski CA, Lombardo F, Dominy BW, Feeney PJ (2001) Experimental and computational approaches to estimate solubility and permeability in drug discovery and development settings. Adv Drug Deliv Rev 64:4-17

27. Kaur H, Singh J, Narasimhan B (2019) Indole hybridized diazenyl derivatives: synthesis, antimicrobial activity, cytotoxicity evaluation and docking studies. BMC Chem 13(1):65

28. Becher R, Wirsel SG (2012) Fungal cytochrome P450 sterol 14a-demethylase (CYP51) and azole resistance in plant and human pathogens. Appl Microbiol Biotechnol 95(4):825-840

29. Rojas JV, López AG, Pérez Y, Cos P, Froeyen M (2019) In vitro evaluation of arylsubstituted imidazoles derivatives as antiprotozoal agents and 
docking studies on sterol 14a-demethylase (CYP51) from Trypanosoma cruzi, Leishmania infantum, and Trypanosoma brucei. Parasitol Res 118(5):1533-1548

30. Ricardo E, Miranda IM, Faria-Ramos I, Silva RM, Rodrigues AG, Pina-Vaz C (2014) In vivo and in vitro acquisition of resistance to voriconazole by Candida krusei. Antimicrob Agents Chemother 58:4604-4611

31. Warrilow AG, Parker JE, Kelly DE, Kelly SL (2013) Azole affinity of sterol 14a-demethylase (CYP51) enzymes from Candida albicans and Homo sapiens. Antimicrob Agents Chemother 57(3):1352-1360

32. Pont A, Williams PL, Loose DS, Feldman D, Reitz RE, Bochra C, Stevens DA (1982) Ketoconazole blocks adrenal steroid synthesis. Ann Intern Med 97(3):370-372

33. Banankhah PS, Garnick KA, Greenblatt DJ (2016) Ketoconazole-associated liver injury in drug-drug interaction studies in healthy volunteers. J Clin Pharmacol 56:1196-1202

34. Kitchen DB, Decornez H, Furr JR, Bajorath J (2004) Docking and scoring in virtual screening for drug discovery: methods and applications. Nat Rev Drug Discov. 3(11):935

35. Meng X-Y, Zhang H-X, Mezei M, Cui M (2011) Molecular docking: a powerful approach for structure-based drug discovery. Curr Comput Aided Drug Des 7(2):146-157

36. Abrigach F, Bouchal B, Riant O, Macé Y, Takfaoui A, Radi S, Oussaid A, Bellaoui M, Touzani R, New N (2016) N, N', N'-tetradentate pyrazoly agents: synthesis and evaluation of their antifungal and antibacterial activities. Med Chem 12(1):83-89
37. Yu L, Lopez A, Anaflous A, El Bali B, Hamal A, Ericson E, Heisler LE, McQuibban A, Giaever G, Nislow C (2008) Chemical-genetic profiling of imidazo $[1,2-a]$ pyridines and-pyrimidines reveals target pathways conserved between yeast and human cells. PLoS Genet 4(11):e1000284

38. Ertl P, Rohde B, Selzer P (2000) Fast calculation of molecular polar surface area as a sum of fragment-based contributions and its application to the prediction of drug transport properties. J Med Chem 43(20):3714-3717

39. Mannhold R, Poda Gl, Ostermann C, Tetko IV (2009) Calculation of molecular lipophilicity: state-of-the-art and comparison of log P methods on more than 96,000 compounds. J Pharm Sci 98(3):861-893

40. Frisch M, Trucks G, Schlegel H, Scuseria G, Robb M, Cheeseman J, Scalmani G, Barone V, Mennucci B, Petersson G: GAUSSIAN09. Gaussian Inc., Wallingford, CT, USA. In.; 2009

41. Grosdidier A, Zoete V, Michielin O (2011) SwissDock, a protein-small molecule docking web service based on EADock DSS. Nucleic Acids Res. 39:W270-W277

42. Pettersen EF, Goddard TD, Huang CC, Couch GS, Greenblatt DM, Meng EC et al (2004) UCSF Chimera - a visualization system for exploratory research and analysis. J Comput Chem 25(13):1605-1612

\section{Publisher's Note}

Springer Nature remains neutral with regard to jurisdictional claims in published maps and institutional affiliations.
Ready to submit your research? Choose BMC and benefit from:

- fast, convenient online submission

- thorough peer review by experienced researchers in your field

- rapid publication on acceptance

- support for research data, including large and complex data types

- gold Open Access which fosters wider collaboration and increased citations

- maximum visibility for your research: over 100M website views per year

At BMC, research is always in progress.

Learn more biomedcentral.com/submissions 